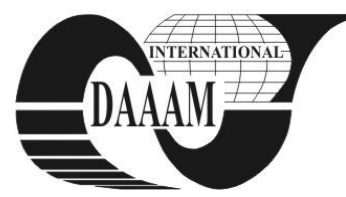

\title{
DETERMINATION OF COMPOSITION AND NATURE OF CRYSTAL THIN SURFACE LAYERS USING X-RAY RADIOGRAPHIC METHOD
}

\author{
MALIQI, R[ashit] \& PLLANA, G[ani]
}

\begin{abstract}
Since the thin layer, created on the semiconductor crystals, has a great impact on the electrical properties, it is important to determine its composition and structure. This analysis has been made on the mono-crystal of cadmium antimony (CdSb), which has a crystalline rhombic net, has 16 atoms in the elementary cells, and crystallises in the group $D_{2 h}^{15}$. After heating the crystal at temperatures higher than 550
\end{abstract}

$K$, significant changes of the electrical features appear, and it has been suspected that structural changes have occurred. With the $x$-ray radiographic method it was concluded that the thin surface of the crystal has different features than the inside of the crystal. This created thin layer is of the order (sequence) $\mu m$ Experimental measurements of electrical quantities that are performed after the creation of this thin layer show that this layer plays a crucial role in the electrical and physical features of the crystal of cadmium antimony. It is concluded that this thin layer has a surplus of antimony, while cadmium evaporated during heating. Detailed measurements of the structural determination of the crystal should be conducted, for which further research is necessary.

Key words: crystal, thin layer, electrical features, temperature

\section{INTRODUCTION}

The research on the physical, chemical and electrical features of the semiconductor materials is of particular importance in the development of nano-electronics and modern technology. The study of stable and meta-stable systems is given a special importance in technique. One such system is the system of antimony cadmium Cd-Sb (Kagan, 1979).

Research undertaken so far, and that are current now, develop in the detection of defects caused by heating of the crystal, radiation with gamma rays, neutrons or other fast particles (Maliqi, 1984).

Experimental measurements show that significant changes occur in the materials, especially in semiconductor crystals during their heating. These appearing changes have been followed during the heating of the crystal up to the temperature of 700 Kelvin. In the previous works, and the measurements (3), we have done during the heating of the crystal, it is concluded that the electrical parameters and its features change during heating at different temperatures. So the specific electrical resistance changes, the Hall's constant and other parameters change as well.

In the recent years the influence of the various radiations on the electrical features of the CdSb system is being examined, in order to be used as a radiation detector.

Experimental measurements show that the various radiations with: neutrons, protons, electrons, gamma rays, etc., form defects in the mono-crystal of cadmium antimony. During this, energy levels occur in the holding zone of $\mathrm{CdSb}$, which are characterized by different effects of recombination. In this way the radiations show changes in the electrical characteristics of CdSb.

In our paper we have examined the impact of the thin layer, created after heating the crystal at temperatures higher than room temperature. During this we have made experimental measurements of the specific electrical conductivity and the Holl's Constants in the crystal p-CdSb, in order of application as a temperature sensor in the detection devices.

\section{EXPERIMENTAL MEASUREMENTS}

Samples for experimental measurements were taken from the mono-crystal p-CdSb. There is a special literature (1), regarding the characteristics (features) of this material. The samples for measurement are of the shape of the parallelepiped (Figure.1) of the crystallographic orientation (001). In these samples we have established four contacts that have been dedicated to the measurement of the electrical parameters. We have painted the surface of the sample with a colour (varnish) to protect it from oxidation during the thermal processing. We have made the electricity contacts $(\mathrm{Ohm})$ on the planes $(001)$ with silver paste and copper conductor. Then we have fitted the sample in a holder with six connection opportunities and we have established the same in a convenient conduit to work with liquid gases, which allows the placing of the sample between the poles of the magnet during the GALVANO magnetic measurements.

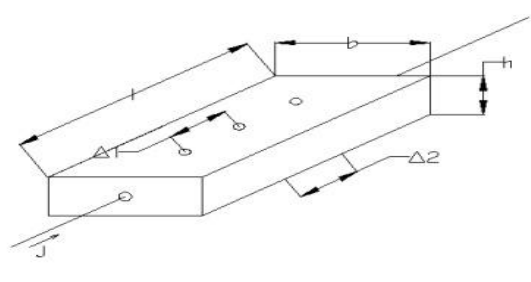

Fig. 1. The shape of the samples which have been used for the experimental measurements

\subsection{Measurement of the specific electric conductivity}

We have measured the specific electrical conductivity with the V-I method, using one-way electricity. The measurement was done starting from room temperature, and when the crystal was heated up to the temperature of $550 \mathrm{~K}$, we have calculated the specific electrical conductivity with the law of Ohm:

$$
\varrho=\frac{l \cdot I}{S \cdot U}
$$

where $S$ - is the surface of the sample, $\ell$ - the samplelength, $\mathrm{U}$ - the tension, and I- the electricity, running through 


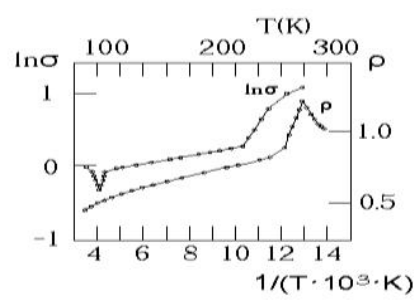

Fig. 2. The current dependency of the specific electrical conductivity and the dependence of the calculation dependency $\ln \sigma$ from the reciprocal value of temperature for $\mathrm{p}$-CdSB

the sample. The current dependency of the specific electrical conductivity is shown in Fig. 2.

\subsection{Temperature measurement of Hall's Constanta}

The measurement of Hall's Constanta is of special importance in the electronics of semiconductors. We have done experimental measurements of the Hall's Constanta on the same prepared sample as for the measurement of specific electrical conductivity.

We have placed the prepared samples through a holder in the container located in the magnetic field. The magnetic field on the surface of the samples was $0.4 \mathrm{~T}$, and had normal direction with the electric field.

Measurements were made for two electricity directions, in order to avoid thermal GALVANO-magnetic effects. The electricity intensity has been $0.5-1 \mathrm{~A}$, in order to avoid the heating of the conductor. We have calculated the Hall's Constanta with the known formula:

$$
R_{H}=\frac{U_{H} \cdot h}{B \cdot I}
$$

where: $U_{H}-$ Hall's tension (voltage), $\mathrm{h}-$ height of sample, B - magnetic field intensity and I - intensity of electricity.

For the measured samples it is shown that the carriers (holders) of the electric loads are the holes, which means that the semiconductor is of the p-type.

Fig. 3. (a) shows the dependence of Hall's Constanta for the samples of the crystal p-CdSb before heating (a), after heating of the crystal at the temperature $\mathrm{T}=545 \mathrm{~K}$ (b) and (c), after the removal of the thin layer, created after heating the crystal.

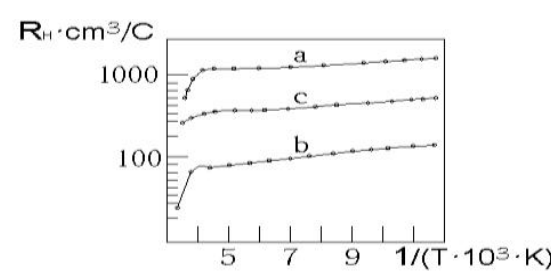

Fig. 3. The temperature dependency of Hall's Constanta on three occasions: a) before heating, b) after heating and c) after removing the thin layer.

\section{DISCUSSION OF THE RESULTS}

In our research, first of all we have concluded that by heating the crystal there appear changes in the resistance, respectively in the specific electric conductivity, and in Hall's Constanta, when heating the crystal.
The results of the measurements of specific conductivity and Hall's Constanta are quite suitable for structural analysis of cadmium antimony. Looking at the obtained curves shows that there are certain intervals, where the specific electrical conductivity is a linear function. The results, obtained by other authors as well as those obtained by us, show that the $\mathrm{CdSb}$ system can be classified as a semiconductor with premise and with a conductivity of the holes.

Heating the crystal at high temperature shows a drastic increase of the specific conductivity and the change of the temperature dependency of Hall's Constanta (see the curves in Fig. 3).

With X-ray methods it has been concluded that during heating the crystal $\mathrm{CdSb}$ at high temperatures, a thin surface layer of the series $\mu$ with a surplus of antimony is created.

After heating the crystal, an x-ray analysis of the nanostructure of the the crystal has been done, with the purpose of identifying its structure after this thermal treatment. The x-ray analyses show the maintenance of the crystal structure, while the created thin layer has different features from the structure of the inside of the crystal.

Further experimental measurements show that after the removal of this thin layer $20 \mu \mathrm{m}$ there is no big difference between the measured quantity values, or they are similar with the measurements before heating.

\section{CONCLUSION}

The obtained results, although incomplete, show that heating the material, or hightemperatures, generate thermal defects in the crystal structure. On the other hand, the results indicate the possibility of practical application of $\mathrm{p}-\mathrm{CdSb}$ as a temperature sensor with linear features in certain temperature intervals. These results indicate that the measurements in this direction require deeper analyses, particularly from the aspect of electronic zonal structure of the $\mathrm{Cd}-\mathrm{Sb}$ system, and the research shouldcontinue in this direction.

\section{REFERENCES}

A. Vol, I.K. Kagan (1979). The structure and Properties of Binary Metallic Unity,Moscow, 1979

Maliqi, R. etc. (1985), ETAN, work.Bulletin, s.XI, p.330, Beograd, 1985

Maliqi, R. (1984). The influence of the higher temperature on the elektrical conductivity of CdSb, ETAN 84, Split Croatia, 1984

Seitz, F. (1979). The Modern Theory of solids, New York, 1979

Wanier, H. etc. (1969). Element of solids state theory, Cambridge University Press, 1969

Ziman M.J. (1972). Principles of the theory of solids, Cambridge University Press, 1972 